PREPARED FOR THE U.S. DEPARTMENT OF ENERGY, UNDER CONTRACT DE-AC02-76CH03073

PPPL-3703

PPPL-3703

UC-70

Hall Thruster Modeling with a Given

Temperature Profile

by

L. Dorf, V. Semenov, Y. Raitses, and N.J. Fisch

June 2002

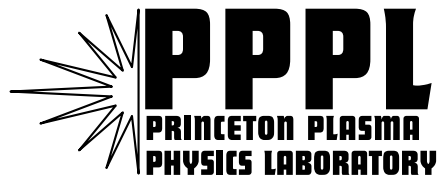

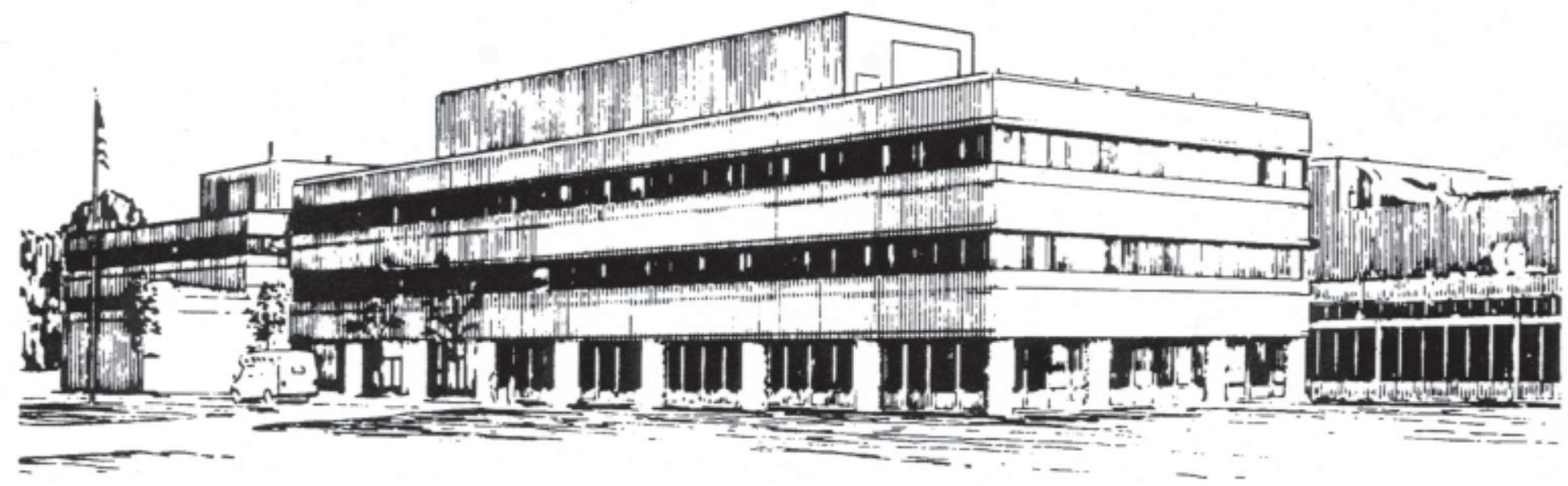

PRINCETON PLASMA PHYSICS LABORATORY PRINCETON UNIVERSITY, PRINCETON, NEW JERSEY 


\section{PPPL Reports Disclaimer}

This report was prepared as an account of work sponsored by an agency of the United States Government. Neither the United States Government nor any agency thereof, nor any of their employees, makes any warranty, express or implied, or assumes any legal liability or responsibility for the accuracy, completeness, or usefulness of any information, apparatus, product, or process disclosed, or represents that its use would not infringe privately owned rights. Reference herein to any specific commercial product, process, or service by trade name, trademark, manufacturer, or otherwise, does not necessarily constitute or imply its endorsement, recommendation, or favoring by the United States Government or any agency thereof. The views and opinions of authors expressed herein do not necessarily state or reflect those of the United States Government or any agency thereof.

\section{Availability}

This report is posted on the U.S. Department of Energy's Princeton Plasma Physics Laboratory Publications and Reports web site in Fiscal Year 2002. The home page for PPPL Reports and Publications is: http://www.pppl.gov/pub_report/

DOE and DOE Contractors can obtain copies of this report from:

U.S. Department of Energy

Office of Scientific and Technical Information

DOE Technical Information Services (DTIS)

P.O. Box 62

Oak Ridge, TN 37831

Telephone: (865) 576-8401

Fax: (865) 576-5728

Email: reports@adonis.osti.gov

This report is available to the general public from:

National Technical Information Service

U.S. Department of Commerce

5285 Port Royal Road

Springfield, VA 22161

Telephone: 1-800-553-6847 or

(703) 605-6000

Fax: (703) 321-8547

Internet: http://www.ntis.gov/ordering.htm 


\title{
Hall thruster modeling with a given temperature profile
}

\author{
L. Dorf, V. Semenov*, Y. Raitses and N.J. Fisch \\ Princeton Plasma Physics Laboratory (PPPL), Princeton, NJ 08543 \\ * Institute of Applied Physics (IPFRAN), Nizhny Novgorod, Russia
}

A quasi one-dimensional steady-state model of the Hall thruster is presented. For given mass flow rate, magnetic field profile and discharge voltage the unique solution can be constructed, assuming that the thruster operates in one of the two regimes: with or without the anode sheath. It is shown that for a given temperature profile the applied discharge voltage uniquely determines the operating regime: for discharge voltages greater than a certain value, the sheath disappears. That result is obtained over a wide range of incoming neutral velocities, channel lengths and widths and cathode plane locations. A good correlation between the quasi 1-D model and experimental results can be achieved by selecting an appropriate temperature profile. We also show how the presented model can be used to obtain a two-dimensional potential distribution.

\section{Introduction}

Since the introduction of the main concept in the late 1950-s by Morozov ${ }^{1}$ and Zharinov, various numerical models were proposed to describe physical processes in $\mathrm{HT}^{2-9}$ To provide the existence and uniqueness of the solution in 1-D or quasi 1-D (considering wall losses) modeling with a given temperature profile one needs to introduce 3 boundary conditions. Since the applied discharge voltage is given, one needs to introduce 2 additional physical constraints on the free parameters.

Fruchtman and Fisch proposed in Ref. [10] that the requirement of sonic transition point, in which the ion velocity, $V_{i}$, equals to the sound velocity, $V_{s}$, to be regular can be used to determine one of the free parameters. To complete a model they assumed zero ion flow at the anode. Later Ahedo et al. in Ref. [2] assumed the presence of the back ion flow at the anode, $z=0$, and changed the condition $V_{i}(0)=0$ to $V_{i}(0)=-V_{s}$, which appears to be more appropriate and results in a physically valid solution over a wide range of discharge voltages. However, we show in this paper that for discharge voltages greater than a certain value this boundary condition also appears to be inappropriate.

In our model we introduce the boundary conditions, which result in the unique solution for all discharge voltages that are typically employed. For the same discharge voltage the Hall thruster may operate in one of the two regimes - with and without the anode sheath. If there is a sheath then, like in Ref [2], we obtain $V_{i}(0)=-V_{s}$. The electron velocity, $V_{e}$, in this case must be selected in order to obtain a total voltage drop in plasma equal to a given $V_{d}$. If there is no sheath then $V_{e}(0)=-V_{\max }$, where $V_{\max }$ is determined only by the electron distribution function at the anode. A given $V_{d}$ in this case determines the ion velocity. We resolve this indeterminacy numerically and show that for discharge voltages greater than a certain value anode sheath disappears. Thus, for a given temperature profile the applied discharge voltage uniquely determines the operating regime and therefore the type of the boundary conditions for $V_{i 0}$ and $V_{e 0}$.

We make use of this approach to obtain the solution for the thruster channel and up to the cathode over a wide range of discharge voltages. We first use a simplified approach to certain issues namely, electron mobility and electron temperature profile, to focus the main attention on boundary conditions. But we also show how these issues can be resolved in order to construct a solution, which correlates well with experimental results for different mass flow rates and discharge voltages. To compare to experiment, we use the data obtained for the Princeton Plasma Physics Laboratory (PPPL) Hall thruster. ${ }^{12}$ The calculated thruster performances namely, thrust, efficiency and propellant utilization, also appear to be in an agreement with experiment. Then we use a 1-D potential profile as a boundary condition on a channel median and solve a quasi 1-D problem in a radial direction to obtain a 2-D potential distribution.

This paper is organized as follows. In Sec. II we set up a physical problem and present a governing system of equations. In Sec. III we describe the boundary conditions indeterminacy and present a numerical procedure for determining the 
operating regimes and the free parameters. In Sec. IV we discuss numerically obtained solutions and present a new approach to determining a temperature profile using experimental data. In Sec. V we discuss "sheath" and "no sheath" regimes. We conclude in Sec. VI with obtaining a 2-D potential distribution.

\section{Quasi 1-D model}

Consider the conventional case of a HT with ceramic channel. The input parameters for any model of the HT are the experimentally controlled parameters, namely, the discharge voltage, $V_{d}$, the propellant mass flow rate, $\frac{d m}{d t}$, and the radial magnetic field profile, $B_{r}(z)$. We neglect the influence of the axial component of the magnetic field. The output parameters to be determined are the discharge current, $I_{d}$, the propellant utilization, and the profiles of ion velocity, ion density and potential.

To describe a steady-state operation of a Hall Thruster we consider the following physical processes. Single ionization: ions are born with the neutral velocity; wall losses: averaged over the channel cross-section; ion acceleration: toward the cathode, use hydrodynamic momentum equation for a mono-energetic ion flow with the ion velocity $V_{i}$; closed electron drift: azimuthal, in $E_{z} \times B_{r}$ direction; electron diffusion: toward the anode, with the electron flow velocity $V_{e}$; free neutral motion: assume the mono-energetic neutral flow with the constant neutral velocity $V_{a 0}$. We also make a quasineutrality assumption: $n_{i}=n_{e}=n$, which is typical for a HT modeling.

In most of our numerical simulations we used input parameters typical for the PPPL Hall thruster operation: ${ }^{12} \quad V_{d}=150-300 \mathrm{~V}$, $\frac{d m}{d t}=1.7-3.0 \mathrm{mg} / \mathrm{s} \quad$ (propellant gas - Xenon), and $B_{\max } \sim 130 G s$. We used the analytical fit consisting of six gauss-functions for magnetic field profile near the channel median as $B_{r}(z)$ (Fig. 1.)

In a 1-D description of a problem it is also necessary to select a distance from the anode, $L_{c}$, at which the voltage drop equals to $V_{d}$, in other words make a choice of a cathode plane. We choose it to be the plane, where the cathode tip is physically located, $L_{c}=5.4 \mathrm{~cm}$ for the PPPL HT. This issue is discussed in a greater detail in Ref [22].

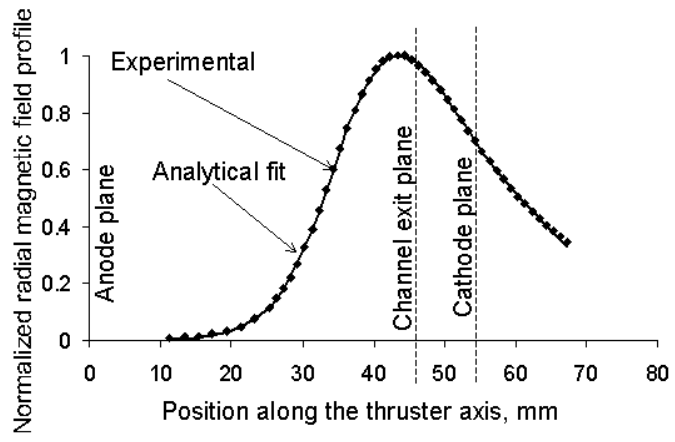

FIG. 1. Normalized magnetic field profile near the channel median for the PPPL HT

In our quasi 1-D model all vectors are projected on to the $z$-axis, where $z$ is the coordinate along the thruster axis, with $z=0$ at the anode. The physical processes can be expressed mathematically as follows:

\section{ION CONTINUITY EQUATION}

$$
\begin{aligned}
& \left(n V_{i}\right)^{\prime}=<\sigma V>n_{a} n- \\
& \frac{2}{H_{c h}} \times 0.55 n \sqrt{\frac{T_{e}}{M_{i}}} \times \Theta\left(L_{c h}-z\right)
\end{aligned}
$$

where the prime sign denotes the derivative with respect to $z$. In the first term of equation (1), the ionization constant, $<\sigma V>\left(T_{e}\right)$, was obtained using experimental data for ionization cross-section, $\sigma_{i}{ }^{X e}\left(E_{e}\right){ }^{13}$ The electron distribution function was assumed to be Maxwellian with the local temperature $T_{e}$, and then the analytical approximation for $\langle\sigma V\rangle\left(T_{e}\right)$ was deduced. In the second term $H_{c h}$ and $L_{c h}$ are the width and the length of a channel respectively $\left(H_{c h}=1.8 \mathrm{~cm}\right.$, $L_{c h}=4.6 \mathrm{~cm}$ for PPPL HT); theta function, $\Theta\left(L_{c h}-z\right)$, represents the absence of the wall losses outside of the channel; and the factor of two indicates the presence of two channel walls. The factor of 0.55 was obtained by solving the radial sheath problem in the hydrodynamic description with the ionization and without collisions, as described by Reimann in Ref [14], however not assuming the quasineutrality in the presheath. 
$\left(n V_{i} V_{i}\right)^{\prime}=\frac{e E n}{M_{i}}-\frac{2}{H_{c h}} \times 0.55 n \sqrt{\frac{T_{e}}{M_{i}}} \times$

$\Theta\left(L_{c h}-z\right) \times V_{i}+<\sigma V>n_{a} n V_{a 0}$

where $E$ is the axial projection of the electric field and $n_{a}$ is the neutral density.

\section{CHARGE CONSERVATION}

$-n V_{e}+n V_{i}=J_{d}$,

where $J_{d}=\frac{I_{d}}{e A_{c h}}, \quad e$ is electron charge, and $A_{c h}=40.7 \mathrm{~cm}^{2}$ is the channel cross-section.

\section{ELECTRON MOMENTUM EQUATION}

$-e n \mu_{e}{ }^{-1} V_{e}=e E n+\left(n_{e} T_{e}\right)^{\prime}$,

We describe the electron axial motion with the phenomenological electron momentum equation, (4), in which $\mu_{e}$ is the absolute value of the electron axial mobility in a radial magnetic field. ${ }^{1,9}$ For the purposes of this paper it is sufficient to assume Bohm diffusion, i.e. $\mu_{e}=\mu_{e}^{\text {Bohm }}=\frac{1}{16 B_{r}(z)}$. However, in order for numerical simulations to be in an agreement with experiment, $\mu_{e}$ must be chosen more carefully, as we show later in this paper.

\section{MASS CONSERVATION}

$n_{a} V_{a 0}+n V_{i}=J_{a 0}+J_{i 0}$,

where $J_{a 0}$ and $J_{i 0}$ are neutral and ion fluxes at the anode respectively. We consider that no ions are coming out of the anode, and all ions hitting the anode recombine with electrons and return to the discharge as neutrals. We therefore obtain: $J_{a 0}=J_{m}-J_{i 0}$, where $J_{m} \stackrel{d e f}{=} \frac{d m / d t}{M_{i} A_{c h}}$ is the propellant flux. In simulations we consider a free molecular neutral flow out of the hot anode $\left(T_{\text {anode }}=1000^{\circ} \mathrm{C}\right)$ to obtain $V_{a 0}=113 \mathrm{~m} / \mathrm{s}$.
$T_{e}(z)=$ Const

Let us first consider a case of constant electron temperature. It is known from experiments, that $T_{e} \sim 3-5 e V$ near the anode, and $T_{e} \sim 18-20 e V$ in the maximum of the temperature profile, ${ }^{15}$ so we choose $T_{e}$ from that interval in our numerical simulations. Later in this paper we return to the question of determining the temperature profile.

\section{Boundary Conditions}

The above system of equations can be reduced to the system of two ordinary differential equations for density, $n(z)$, and ion flux, $J_{i}(z)=n(z) V_{i}(z)$ :

$$
\left\{\begin{array}{c}
J_{i}^{\prime}=n<\sigma V_{e}>\left(T_{e}\right) \frac{J_{m}-J_{i}}{V_{a 0}}-1.1 \frac{n V_{s}}{H_{c h}} \\
n^{\prime}=\frac{1}{1-V_{i}^{2} / V_{s}^{2}}\left[\frac{e}{M_{i} \mu_{e} V_{s}} \frac{J_{d}-J_{i}}{V_{s}}-\right. \\
n\left(\left(\ln T_{e}\right)^{\prime}-\frac{<\sigma V_{e}>\left(T_{e}\right)}{V_{s}} \frac{J_{m}-J_{i}}{V_{s}}\right)- \\
\left.\frac{J_{i}}{V_{s}}\left(2 \frac{<\sigma V_{e}>\left(T_{e}\right)}{V_{s}} \frac{J_{m}-J_{i}}{V_{a 0}}-\frac{1.1}{H_{c h}}\right)\right],
\end{array}\right.
$$

where $V_{s}=\sqrt{\frac{T_{e}}{M_{i}}}$ is the ion acoustic velocity. The $\Theta$-function in terms originating from the walllosses term in (1) was omitted for simplicity.

If the ion flux and the plasma density at the anode and the charge flux, $J_{d}$, are specified, one can try to integrate (7) numerically. In other words, the system (7) contains three free parameters to be determined before the solution can be obtained: $n_{0}$, $M_{0}$ and $\bar{V}_{0}$, where $M=\frac{V_{i}}{V_{s}}, \bar{V}=\frac{V_{e}}{V_{t e}}, V_{t e}=\sqrt{\frac{T_{e}}{m_{e}}}$ is the electron thermal velocity, and the subscript "naught" means that functions are evaluated at the anode, $z=0 . M_{0}$ and $\bar{V}_{0}$ explicitly enter in the charge flux, $J_{d}=n_{0}\left(-\bar{V}_{0} V_{t e 0}+M_{0} V_{s 0}\right)$, and the ion flux at the anode, $J_{i 0}=n_{0} M_{0} V_{s 0}$. Thus, one needs three boundary conditions to provide the existence and uniqueness of the solution. 
Neglecting wall losses in the ion continuity equation and assuming $V_{a 0}=0$ in the ion momentum equation, in order to better demonstrate our approach to determining the free parameters, we can deduce the following normalized equation for ion Mach number:

$d_{t} M=\frac{\left(1+M^{2}\right)\left(1-J_{i} / J_{m}\right) \beta-M^{2}\left(J_{d} / J_{i}-1\right) \gamma}{1-M^{2}}$

where $\quad t \stackrel{\text { def }}{=} z / H_{c h}, \quad \beta \stackrel{\text { def }}{=} \frac{<V>\left(T_{e}\right) J_{m} H_{c h}}{V_{a 0} V_{s}}$, $\gamma=\frac{d e f}{V_{s}}$, and $\omega_{B i}=\frac{e B_{r}}{M_{i}}$ is the ion cyclotron frequency.

The equation (8) describes the ion dynamics in a quasineutral plasma. A similar equation describes the flow dynamics in the well-known de Laval nozzle. ${ }^{16}$ Other authors ${ }^{2,10}$ also dealt with this type of equation however, we find that there is still a need for additional analysis. The first (positive) term in the numerator of (8) originates from the ionization term in (1) and leads to ion acceleration in the subsonic region of the ion flow, i.e. where $M<1$. The second (negative) term in the numerator of (8) is originally the electric field term from the equation (2), and it effectively works in subsonic plasma as an ion drag. The denominator appears essentially because of the electron pressure and it turns to zero at the boundary of the subsonic flow, at which $M=1$. This leads to a singularity, typical for quasineutral plasmas and called the "sonic transition". ${ }^{16} \mathrm{We}$ look only for a non-singular solution of system (7) which describes a smooth behavior of all physical values in the vicinity of the sonic transition point, $z_{s t}$, at which $M=1$. Fruchtman and Fisch in Ref. [10] considered the possibility of abrupt sonic transition in HT with an additional electrode placed inside the channel, and Ahedo et al in Ref. [2] proposed a "choked-exit" type of solution, in which ions reach the sound velocity right at the channel exit. However, all of the authors considered a smooth sonic transition in their models of the conventional Hall Thrusters.

As can be seen from (8), in order for the sonic transition point to be regular it is necessary that the drag and acceleration terms are equal at this point. Both of these terms depend on $J_{d}$ and $J_{i 0}$, i.e. on the free parameters that are set at $z=0$. Out of the three free parameters, only $n_{0}$ and $M_{0}$ enter explicitly in both $J_{d}$ and $J_{i 0}$, and, as will be shown in the next paragraph, $M_{0}$ and $\bar{V}_{0}$ are physically interdependent. So, one can conclude that exactly the choice of $n_{0}$ is responsible for the smooth sonic transition. We set $M_{0}$ and $\bar{V}_{0}$ in the interval $[0,1]$ and tried to select $n_{0}$ numerically in order to obtain a non-singular (NS) solution. It was shown by a comprehensive scanning over all reasonable for HT values of $n_{0}$, that a smooth sonic transition takes place only if $n_{0}$ equals to a certain unique value, $n_{0}{ }^{N S}$, which depends, of course, on $\left(\bar{V}_{0}, M_{0}\right)$. If $n_{0}>n_{0}{ }^{N S}$, the drag term appears to be too big and $M$ does not reach 1 anywhere in the channel; and if $n_{0}>n_{0}{ }^{N S}$, the drag term is too small and a numerator in (8) appears to be greater than zero at $z_{s t}$, which leads to a singularity (Fig. 2). Thus, for given $\left(\bar{V}_{0}, M_{0}\right)$ the requirement of the sonic transition point to be regular results in the unique value of $n_{0}$.
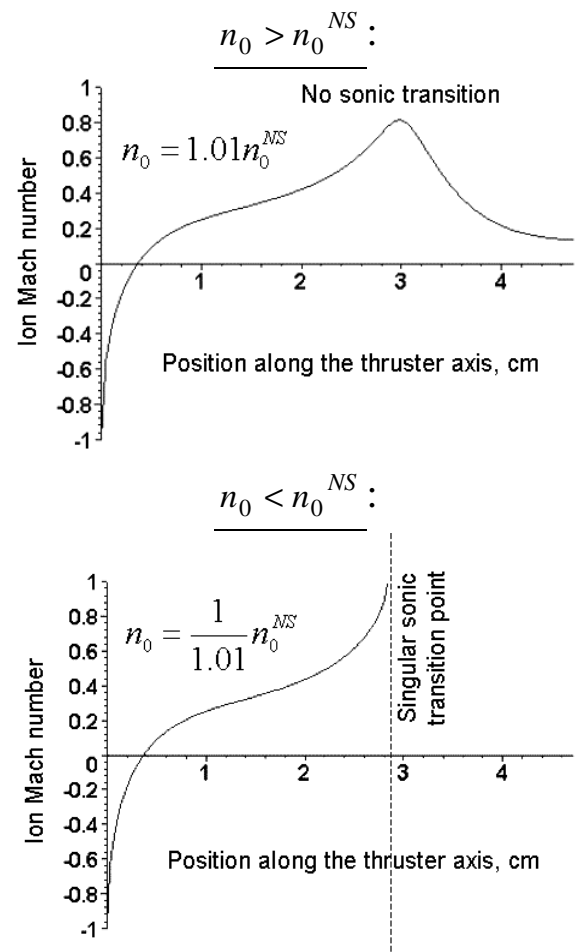

FIG. 1. Types of the ion velocity spatial behavior for different values of the plasma density at the anode. Case $T_{e}=$ Const $\mathrm{T}$

There are two possibilities in determining $\bar{V}_{0}$ and $M_{0}$. For the same discharge voltage the Hall thruster as every gas discharge may operate in one of the two regimes - with and without the anode sheath. If there is a sheath then, like in Ref [2], we 
obtain that $M_{0}=-1$. The electron velocity, $\bar{V}_{0}$, in this case must be selected in order to obtain a total voltage drop in plasma equal to a given $V_{d}$ : $\int_{0+}^{L c} E(z) d z=V_{d}$, where $L_{c}$ is the distance from the anode to the cathode plane, and " $0+$ " means that the integration must be produced only over quasineutral plasma (we neglected a sheath voltage drop here, because $T_{e}$ at the anode is usually very small in a real HT). If there is no sheath and plasma is quasineutral up to the anode, then $\bar{V}_{0}=-\bar{V}_{\max }$, where $\bar{V}_{\max }$ is determined only by the electron distribution function at the anode (we used $\bar{V}_{\max }=0.4$ in our simulations, assuming Maxwellian distribution). In this case ions accelerated in the presheath toward the anode do not reach the sound velocity and $M_{0}$ is determined by a given $V_{d}$.

To resolve this indeterminacy we numerically scanned in the $\left(\bar{V}_{0}, M_{0}\right)$ plane along the physically possible curve, as shown on Fig. 3. We found that $V_{d}$ and $I_{d}$ monotonically grow as we gradually transfer from "sheath" to "no sheath" regime. For $V_{d}>V_{d}{ }^{*}$, where $V_{d}{ }^{*}$ corresponds to the point (- $\bar{V}_{\max }$, $-1)$ in the $\left(\bar{V}_{0}, M_{0}\right)$ plane, there is no anode sheath. So, for given $T_{e}$ and $\mu_{e}$ the discharge voltage, $V_{d}$, uniquely determines the operating regime and $\left(\bar{V}_{0}, M_{0}\right)$. The boundary condition issue is resolved.

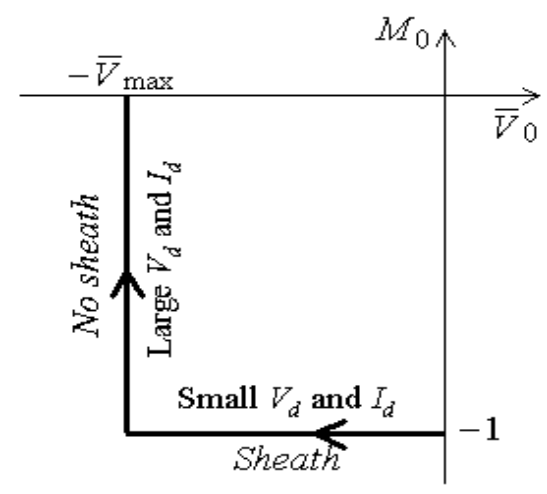

FIG.3. A physically possible curve in the $\left(M_{0}, \bar{V}_{0}\right)$ crosssection of the free parameters space.

\section{Solution}

The above boundary conditions were used to determine the free parameters and obtain solutions with several constant temperatures. It was found that, like in some other models, ${ }^{10}$ at large temperatures all of the propellant is ionized in a very short region near the anode, and at the smaller temperatures the propellant utilization, $\frac{J_{i}\left(L_{c h}\right)}{J_{m}}$, appears to be atypically small for a HT. At temperatures smaller than a certain lower threshold, ionization appears to be insufficient for normal operation of the thruster and it becomes impossible to build a non-singular solution with supersonic ion velocity at the thruster exit with any free parameters.

It was shown that the same approach to determining the free parameters can be applied in the case of any given shape of temperature profile, qualitatively similar to experimental, ${ }^{15}$ if maximal temperature, $T_{\max }$, is chosen to be large enough (see Appendix). We investigated the dependence of the solution on the shape of the temperature profile, and for each considered point $\left[V_{d}, J_{m}, B_{r}(z)\right]$ in the typical PPPL HT operational range were able to determine $T_{e}(z)$ that results in the experimental value of $I_{d}$ and $\frac{n_{\max }}{n_{0}} \sim 10$, which is typical for HT. ${ }^{15}$ From the same argument we have determined the actual value of electron mobility: $\mu_{e} \sim(1 / 8-$ 1/6) $\mu_{e}{ }^{B o h m}$. The fact that electron mobility in Hall thrusters appears to be several times less than the one obtained with a Bohm diffusion concept was also discovered by some other authors. ${ }^{8,17,18}$

The numerically obtained profiles for $V_{d}=$ $240 \mathrm{~V}$ and a mass flow rate of $1.7 \mathrm{mg} / \mathrm{s}$ are shown on Fig. 4. It was found that $M_{0}=-1$ and $\bar{V}_{0}=-0.06$ for such input parameters, so the thruster operates in a "sheath" regime. The propellant utilization (about 86\%) and potential profile were found to be in an agreement with experiment. ${ }^{12,19}$ We have also calculated the thruster performances namely, thrust, $T$, and efficiency, $\eta$, using the following expressions:

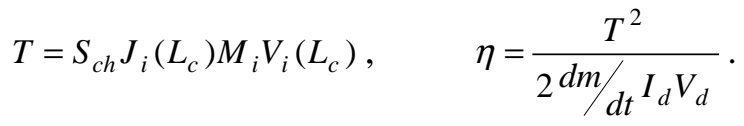

The results also turned out to be in a correlation with experiment: $\quad T=25 \mathrm{mN}, \quad \eta=47 \% .^{12}$ 

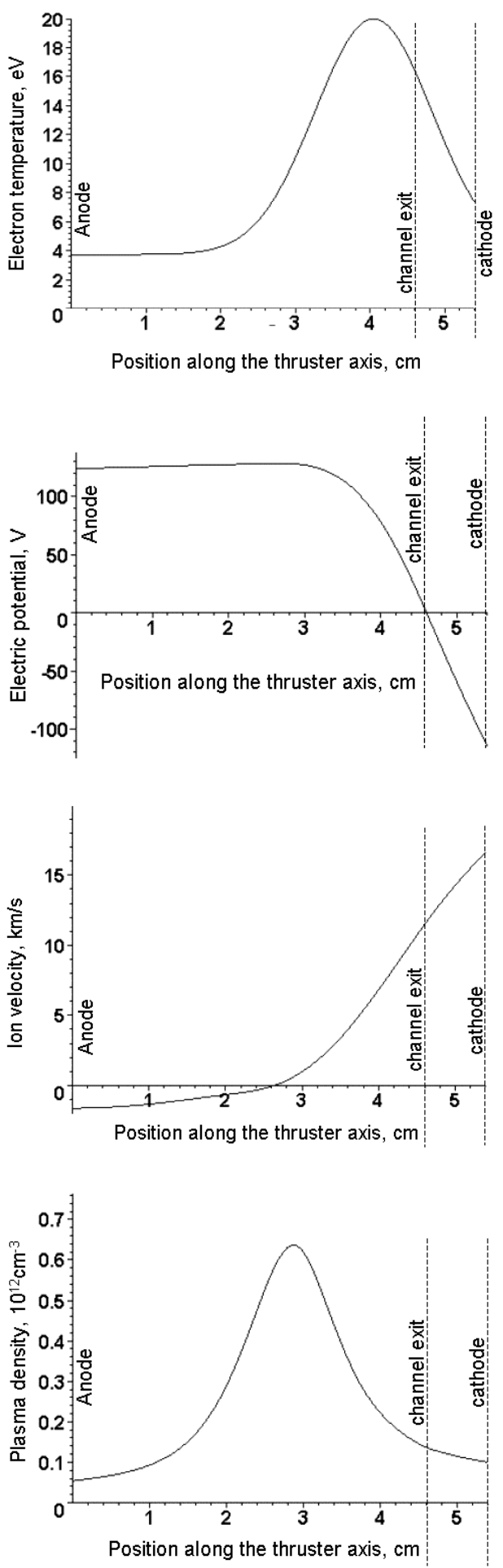

FIG. 4 The numerically obtained profiles in a Hall thruster.

$$
\text { For } V_{d}=240 \mathrm{~V}, \frac{d m}{d t}=1.7 \mathrm{mg} / \mathrm{s} \text {. }
$$

Zero potential was chosen at the channel exit.

$$
z_{s t}=3.52 \mathrm{~cm}, I_{d}=1.63 \mathrm{~A}
$$

\section{Discussions}

The temperature profile found for $V_{d}=240 \mathrm{~V}$ and $\frac{d m}{d t}=1.7 \mathrm{mg} / \mathrm{s}$ was used to obtain solutions for $\frac{d m}{d t}=1.7 \mathrm{mg} / \mathrm{s}$ at several discharge voltages and the same $B_{r}(z)$. The numerically obtained thruster $V-I$ characteristic is presented on the Fig. 5. The $V-I$ characteristics with corresponding $T_{e}(z)$ were also obtained for the mass flow rates of $2 \mathrm{mg} / \mathrm{s}$ and $2.5 \mathrm{mg} / \mathrm{s}$, and for several channel lengths and widths. For most of the considered $\left(V_{d}, J_{m}\right)$ in the typical PPPL HT operational range the anode sheath appeared to take place. So, at moderate discharge voltages $M_{0}=-1$ can be used as a universal $\mathrm{BC}$ for modeling of a HT, as suggested in Ref. 2. As was shown, $\bar{V}_{0}$ in this case must be selected in order to obtain a desired $V_{d}$. The negative ion flux toward the anode was indeed measured in experiments. ${ }^{15}$ But for discharge voltages greater than a certain value, $V_{d}$ *, which increases when the mass flow rate is increased, sheath disappeared and in order to obtain a solution it was necessary to use alternative, "no sheath" type boundary conditions: $\bar{V}_{0}=-\bar{V}_{\max }$, $M_{0}$ is determined by $V_{d}$. The "no sheath" regime was also observed experimentally ${ }^{20}$. It was found that for the same $V_{d}, J_{m}$ and $B_{r}(z)$ an absolute value of $\bar{V}_{0}$ increases and may even reach $\bar{V}_{\max }$ when the channel length from the anode side is artificially decreased. It was also discovered that $V_{d}$ *, separating "sheath and "no sheath" regimes, increases when the electron mobility is increased.

The presented quasi 1-D model with a given temperature profile is, of course, simplified and not completely suitable for a quantitative description of the real thruster behavior in the experiments. However, it proved to be useful for a qualitative analysis and for better understanding of the HT operation in a wide range of input parameters. The main results are recognizing a possibility of the thruster operation without the anode sheath and presenting a method of determining the thruster operating regime and the free parameters for a 1-D modeling. The presented approach involves only global physical phenomena: anode sheath and sonic transition. So, it can be suggested that this approach is applicable for a 2-D modeling, especially considering that 2-D effects take place mainly in the region of a strong magnetic field, far from the 
anode. Let us also notice that the applicability of the described approach to determining the free parameters was shown for different profiles of the magnetic field, qualitatively similar to the one used in a PPPL HT. The authors also believe that the same approach can be applied for modeling of other types of Hall thrusters such as segmented electrode $H T$ and anode layer thruster. ${ }^{12,21}$

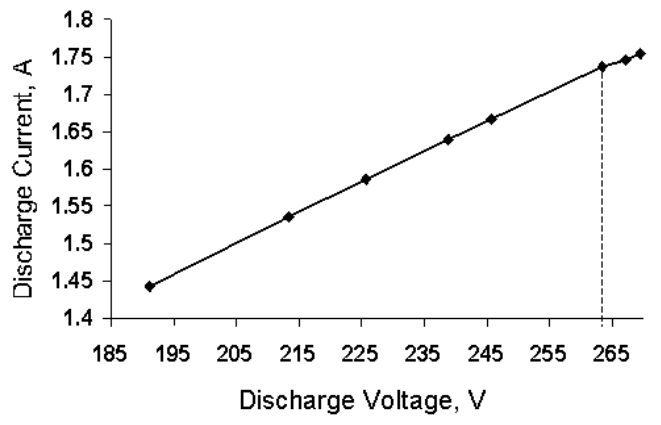

FIG. 5. The numerically obtained Hall thruster V-I characteristic. $\frac{d m}{d t}=1.7 \mathrm{mg} / \mathrm{s}$. For $V_{d}>263 \mathrm{~V}$ there is no anode sheath.

\section{2-D Potential distribution}

A quasi 1-D model can be used to obtain an approximate 2-D potential distribution. Let us consider a planar geometry with $z$-axis along the channel median and $r$-axis in the radial direction. The ion continuity equation and the radial projection of the ion momentum equation then become:

$\frac{\partial J_{z}}{\partial z}+\frac{\partial J_{r}}{\partial r}=<\sigma V>n_{a} n_{e}$

where $J_{z}$ and $J_{r}$ are the axial and the radial projections of the ion flux respectively. We assume for simplicity that $\frac{\partial J_{r}}{\partial r}$ is independent of $r$ and that $J_{r}(z, 0)=0$, i.e. that ion flux on the channel median is purely axial. Then $\frac{\partial J_{r}}{\partial r}=\frac{\Gamma_{\text {wall }}}{H_{c h} / 2}$, where $\Gamma_{\text {wall }}$ is ion flux to the wall. As was already mentioned, it can be shown that $\Gamma_{\text {wall }}=0.55 n(z, 0) \sqrt{\frac{T_{e}(z)}{M_{i}}}$; electron temperature is assumed independent of $r$.
Thus, we obtain:

$\frac{\partial J_{r}}{\partial r}=1.1 \frac{n(z)}{H_{c h}} \sqrt{\frac{T_{e}(z)}{M_{i}}}$,

where $n(z)$ is a plasma density obtained from a quasi 1-D modeling. We assume all the quantities on a channel median to be those found from a quasi 1-D modeling.

Let us introduce the following normalized variables: $\quad t=r / r_{d}, \quad u=e(\varphi-\varphi(z)) / T_{e}(z)$, $e=\partial u / \partial t, \quad N_{e, i}=n_{e, i} / n(z), \quad$ where $r_{d}=\sqrt{T_{e}(z) / 4 \pi n(z) e^{2}}, \varphi$ is electric potential, $\varphi(z)$ is electric potential obtained from a quasi 1-D modeling, and $n_{e, i}$ are the electron and ion densities (we give up a quasineutrality assumption away from the channel median). We assume a Boltzman distribution for electrons:

$N_{e}=\exp (u)$

A quasineutrality assumption made in a 1-D modeling leads to the following form of a Poisson equation:

$\partial e / \partial t=N_{e}-N_{i}$

Using a radial projection of the ion momentum equation: $\quad \frac{\partial\left(V_{z} J_{r}\right)}{\partial z}+\frac{\partial\left(V_{r} J_{r}\right)}{\partial r}=e E_{r} n_{i} / M_{i}$, together with equations (10) - (12) and neglecting $\partial_{z}\left(V_{z} J_{r}\right)$ for simplicity, we can deduce the following approximate expression for the normalized ion density:

$$
N_{i}=\frac{(\lambda t)^{2}}{e^{2} / 2+1-\exp (u)},
$$

where $\lambda=1.1 r_{d}(z) / H_{c h}$.

The equations (11) - (13) can be independently integrated along $r$ in every point on a $z$-axis with a local value of $r_{d}(z)$. Since $u(0)=0$ by definition of $u$, we only need to supply the above system with one more boundary condition on a channel median: $e(z, 0)=0$. The numerically obtained 2-D potential distribution is presented on a Fig. 6. The shape of the equipotentials is found to be in a reasonable agreement with experiment. ${ }^{19}$ 


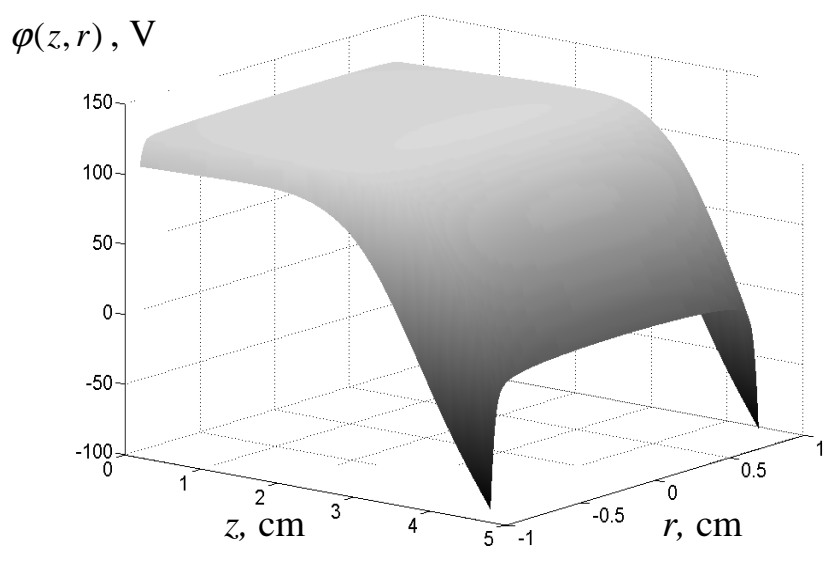

FIG. 6. Numerically obtained 2-D potential distribution. For $V_{d}=240 \mathrm{~V}, \frac{d m}{d t}=1.7 \mathrm{mg} / \mathrm{s}$.

Appendix: Selection of the appropriate $T_{\max }$

As was mentioned, in order for the described approach to determining free parameters to work properly for discharge voltages in the practically used range, $T_{\max }$ for the electron temperature profile, $T_{e}(z)=T_{\max } \cdot \operatorname{Shape}(z)$, must be chosen large enough for a given $\mu_{e}$. Otherwise, as we move in the $\left(\bar{V}_{0}, M_{0}\right)$ plane along the physically possible curve from "Sheath" to "No sheath" region (Fig. 3), we will find the discharge current to very slowly increase, whereas the discharge voltage will significantly decrease. Of course, in the real HT the decrease of discharge voltage at the same magnetic field profile leads to the decrease of a discharge current and, as was shown before, the decrease of $V_{d}$ should occur when we move from "No sheath "to" Sheath region, not on the contrary. It was shown that at the same mass flow rate the minimal value of $T_{\max }$ depends strongly on the incoming neutral velocity. Basically, if $T_{\max }$ for which solution can be constructed with a certain $V_{a 0}$ is known, then in order to be able to construct a solution with another $V_{a 0}, T_{\max }$ must be chosen so that to keep $\frac{<\sigma V>\left(T_{\max }\right)}{V_{a 0}}$ approximately the same.

\section{Acknowledgement}

The authors would like to thank M. Keidar, D. Staack, A. Smirnov and A. Litvak for very useful discussions and their comments on this paper.
This work was partially supported by the US DOE under contract No. DE-AC02-76CH03073 and the Russian Foundation for Basic Research.

\section{References}

1. A. I. Morozov and V. V Savelyev, in the "Reviews of Plasma physics", edited by B. B. Kadomtsev and V. D. Shafranov, vol. 21

2. E. Ahedo, P. Martinez-Cerezo, M. Martinez-Sanches, Phys. Plasmas 8, 3058 (2001)

3. E. Ahedo, P. Martinez-Cerezo, M. Martinez-Sanches, AIAA paper No. 2001-3323, 37-th Joint Propulsion Conference and Exhibit, July 8-11 2001, Salt Lake City, Utah

4. I. Katz, G. Jongeward, V. Davis, M. Mandell, I. Mikellides, R. Dressler, I. Boyd, K. Kannenberg, AIAA paper No. 2001-3355, 37-th Joint Propulsion Conference and Exhibit, July 8-11 2001, Salt Lake City, Utah

5. I. G. Melikidze, I. Katz, M. Mandell, J. S. Snyder, AIAA paper No. 2001-3505, 37-th Joint Propulsion Conference and Exhibit, July 8-11 2001, Salt Lake City, Utah

6. M. Keidar, I. D. Boyd, J. Appl. Phys. 86, 4786 (1999)

7. D. B. VanGilder, I. D. Boyd, M. Keidar, J. Spacecraft Rockets 37, 129 (2000)

8. M. Keidar, I. D. Boyd, and I. I. Beilis, Phys. Plasmas 8, 5315 (2001)

9. K. Makowsky, Z. Peradzynski, N. Gascon, and M. Dudeck, AIAA paper No. 1999-2295, 35-th Joint Propulsion Conference and Exhibit, June 20-24 1999, Los Angeles, California

10. A. Fruchtman, N. J. Fisch and Y. Raitses, Phys. Plasmas 8, 1048 (2001)

11. V. I. Baranov, Y. S. Nazarenko, V. A. Petrosov, A. I. Vasin, IEPC paper No. 99-103, 26-th International Electric Propulsion Conference, Oct. 17-21, 1999, Kokura-kita, Kitakyushu, Japan

12. Y. Raitses, L. A. Dorf, A. A. Litvak, and N. J. Fisch, J. Appl. Phys. 88, 1263 (2000)

13. D. Rapp and P. Englander-Golden, J. Chem. Phys. 43, 1464 (1965)

14. K-U Riemann, J. Phys. D: Appl. Phys. 24, 493 (1991)

15. A. M. Bishaev and V. Kim, Sov. Phys. Tech. Phys. 23, 1055 (1978)

16. F. I. Frank, Izvestia Akademii Nauk SSSR, Ser. Matematika, vol. IX, 1945

17. E. Ahedo, P. Martinez, and M. Martinez-Sanches, AIAA paper No. 2000-3655, 36-th Joint Propulsion Conference, Huntsville, AL

18. J. M. Fife and S. Locke, AIAA paper No. 2001-1137, 39th Aerospace Sciences Meeting and Exhibit, Reno, NV, Jan. 8-11, 2001

19. Y. Raitses, M. Keidar, D. Staack and N. J. Fisch, J. Appl. Phys., submitted Nov. 2001

20. A. I. Bugrova and V. Kim, in "Plasma Accelerators and Ion Injectors" (Nauka, Moscow, 1984) 107 (in Russian)

21. V. V. Zhurin, H. R. Kaufman and R. S. Robinson, Plasma Sources Sci. Technol., 8, R1-R20 (1999) (printed in the UK)

22. L. Dorf, V. Semenov, Y. Raitses, N.J. Fisch, submitted to Phys. Plasmas, Mar, 2002 


\section{External Distribution}

Plasma Research Laboratory, Australian National University, Australia

Professor I.R. J ones, Flinders University, Australia

Professor J oão Canalle, Instituto de Fisica DEQ/IF - UERJ , Brazil

Mr. Gerson O. Ludwig, Instituto Nacional de Pesquisas, Brazil

Dr. P.H. Sakanaka, Instituto Fisica, Brazil

The Librarian, Culham Laboratory, England

Library, R61, Rutherford Appleton Laboratory, England

Mrs. S.A. Hutchinson, JET Library, England

Professor M.N. Bussac, Ecole Polytechnique, France

Librarian, Max-Planck-Institut für Plasmaphysik, Germany

J olan Moldvai, Reports Library, MTA KFKI-ATKI, Hungary

Dr. P. Kaw, Institute for Plasma Research, India

Ms. P.J . Pathak, Librarian, Insitute for Plasma Research, India

Ms. Clelia De Palo, Associazione EURATOM-ENEA, I taly

Dr. G. Grosso, Instituto di Fisica del Plasma, Italy

Librarian, Naka Fusion Research Establishment, J AERI, J apan

Library, Plasma Physics Laboratory, Kyoto University, J apan

Research Information Center, National Institute for Fusion Science, J apan

Dr. O. Mitarai, Kyushu Tokai University, J apan

Library, Academia Sinica, Institute of Plasma Physics, People's Republic of China

Shih-Tung Tsai, Institute of Physics, Chinese Academy of Sciences, People's Republic of China

Dr. S. Mirnov, TRINITI, Troitsk, Russian Federation, Russia

Dr. V.S. Strelkov, Kurchatov Institute, Russian Federation, Russia

Professor Peter Lukac, Katedra Fyziky Plazmy MFF UK, Mlynska dolina F-2, Komenskeho Univerzita, SK-842 15 Bratislava, Slovakia

Dr. G.S. Lee, Korea Basic Science Institute, South Korea

Mr. Dennis Bruggink, Fusion Library, University of Wisconsin, USA

Institute for Plasma Research, University of Maryland, USA

Librarian, Fusion Energy Division, Oak Ridge National Laboratory, USA

Librarian, Institute of Fusion Studies, University of Texas, USA

Librarian, Magnetic Fusion Program, Lawrence Livermore National Laboratory, USA

Library, General Atomics, USA

Plasma Physics Group, Fusion Energy Research Program, University of California at San Diego, USA

Plasma Physics Library, Columbia University, USA

Alkesh Punjabi, Center for Fusion Research and Training, Hampton University, USA

Dr. W.M. Stacey, Fusion Research Center, Georgia Institute of Technology, USA

Dr. J ohn Willis, U.S. Department of Energy, Office of Fusion Energy Sciences, USA

Mr. Paul H. Wright, Indianapolis, Indiana, USA 
The Princeton Plasma Physics Laboratory is operated by Princeton University under contract with the U.S. Department of Energy.

\author{
Information Services \\ Princeton Plasma Physics Laboratory \\ P.O. Box 451 \\ Princeton, NJ 08543
}

Phone: 609-243-2750

Fax: 609-243-2751

e-mail: pppl_info@pppl.gov

Internet Address: http://www.pppl.gov 